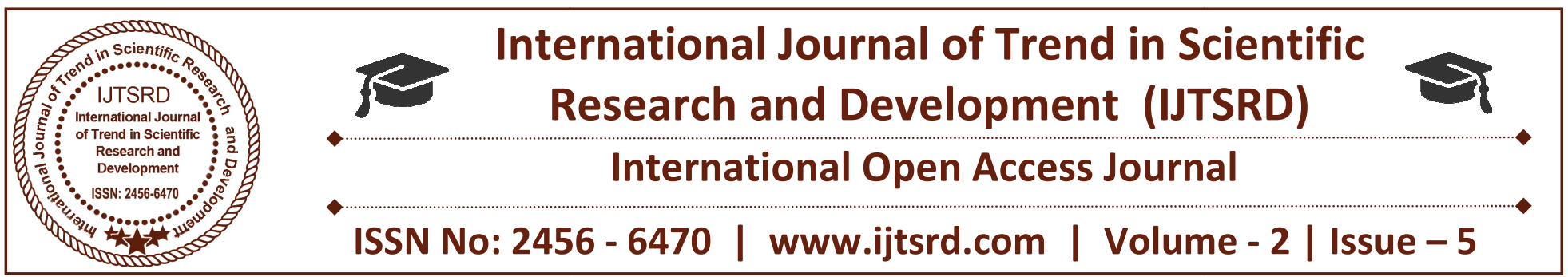

\title{
Hand Rehabilitation Glove
}

\author{
Raja Vikram Reddy ${ }^{1}$, Aliasgar Barodawala ${ }^{2}$ \\ ${ }^{1}$ Professor, ${ }^{2}$ Student, ${ }^{1,2}$ M H Saboo Siddik College of Engineering, Mumbai University, \\ New Nagpada, Mazgaon, Mumbai, Maharashtra, India
}

\begin{abstract}
In this paper, we present the mechanism which will be helpful for an individual to cope up with the Rehabilitation exercises. Mechanism proposed here will be useful for assisting human being in case of the Hand Rehabilitation. We propose to design a pair of soft wearable hand glove which comfortably fits on the human hand. One hand will be the Motion detection Glove which includes the Flex Sensors and the other is the assistive hand used for performing the Rehabilitation exercises. Basically our designed glove could be used for robotic orthosis.
\end{abstract}

Keywords: Hand Rehabilitation; Flex Sensor; Motion Detection Glove; Exoskeletal Glove; Robotic Orthosis.

\section{INTRODUCTION}

From a purely physiological point of view, the hand is entirely engineered device comprised of 27 bones, 24 muscles, and 32 joints [1].

The term robot is often used but seldom understood. A robot may be broadly defined as a machine that is in some way physically similar to a human or that performs a function similar to that performed by a human [2].

Hand Structures are made up of dense connective tissue and the ability of this strong dense connective tissue to glide in relation to one another is essential for the functioning of the normal Hand. Phalange al motor impairment is the most common condition found in the patients suffering from the Accidental fractures or neurological disarray. The patients who are lacking motor skills undergo into traditional physical therapy training in which they are provided To perform various repetitive tasks to regain the original motor skills as that of original,

The intended use of our mechanism is to provide physiotherapy exercises to the person who recently met hand fracture and is in a need of physiotherapy exercises. In general, the exoskeleton hand will receive motor action commands from the human user. The movements of the robotic palm are controlled by moving the user's fingers using the Flex sensors and Bluetooth modules which is the IEEE 802.15 protocol in use. Bluetooth module provides range up to 10 meters (LOS).

The project aims to design and implement a costeffective and an affordable prototype model of robotic hand for rehabilitation. The flex sensor system gives control signals to the arm wirelessly via Bluetooth and hand mimics the movement of the flex sensor system.

\section{BLOCK DIAGRAM}

The system consists of two subdivisions, the Master $\&$ the Slave Section. The Block Diagram for the same is depicted in the Figure 1. Transmitter Section includes the Flex sensors connected to the transmitting ATmega328 along with the Bluetooth HC-05 Bluetooth wireless module. Receiver section includes the Servo motors connected with receiver ATmega328 along with receiver Bluetooth module [4] 


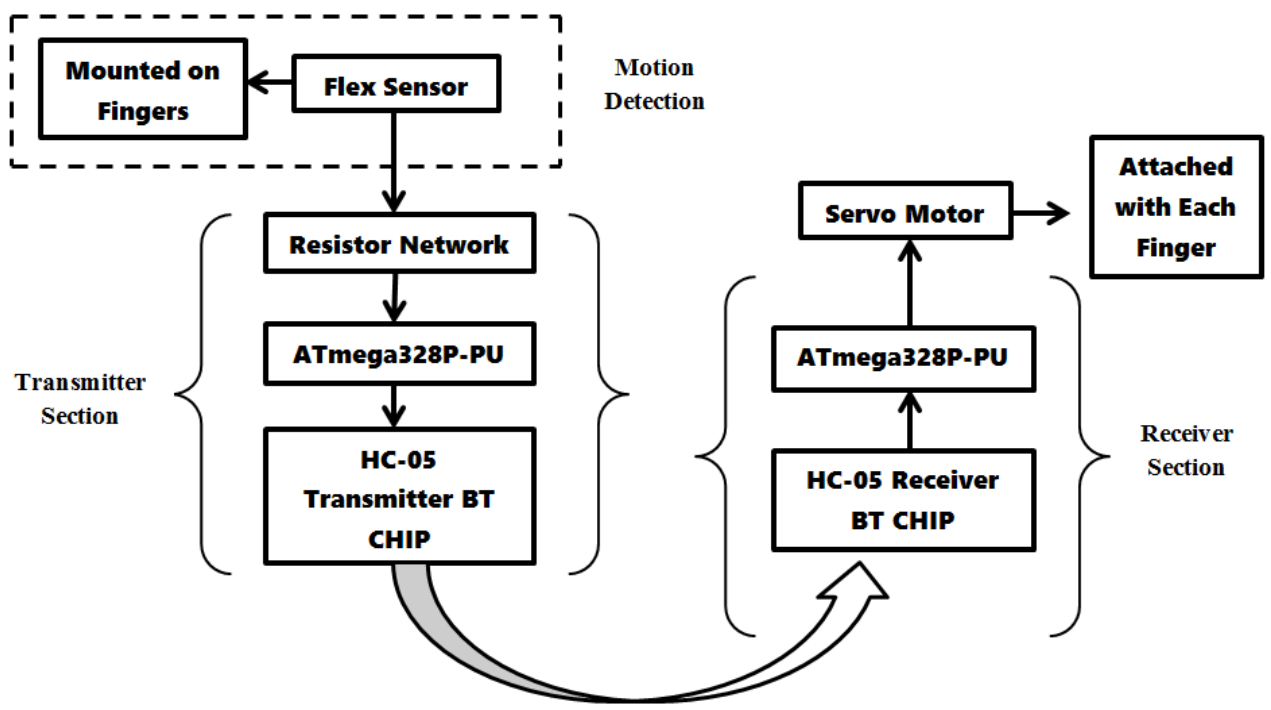

Figure 1: Block Diagram of Hand Rehabilitation Glove.

\section{A. MASTER SECTION}

This section acts as a transmitter section. This section includes flex sensors which will be equipped on a transmitting glove. As from the Figure 1, flex sensors will be mounted on each finger of the hand. When bends or force is applied on the flex sensors it will generate the analog signal of each force being applied. These signal then processed in the ATmega328. These signal is then transmit to the receive side by using Bluetooth hc-05 module

\section{B. SLAVE SECTION}

Slave section includes the Servo motors. The signal which is sent from Master side is received by using Receiver Bluetooth HC-05. As shown in the Figure 1 signal is then fed to the ATmega328. According to the value it will control the servo motors. Each servo motor is connected with the each finger \& according to the signal each finger will move and the replica of the movement which will be done at the transmitter side will be cloned at the receiver side.

\section{Designing The Motion Detection Glove}

\section{A. Mounting Flex sensors}

The flex sensors are used to detect the motion of each individual finger. Onto each phalange flex sensor is placed in the state of equal spaces from the end. Flex Sensor generates different values for the different degree of bend.

\section{B. Mounting Resistor Network}

In order to generate the difference signals, a voltage divider needs to be applied. Five Voltage divider Networks were designed accordingly

\section{Voltage Divider Network}

Variations in the Values of resistance are needed, so a variable Resistor i.e. Flex sensor with a combination of fixed resistor of $10 \mathrm{~K}$ connected in series was used, the variations in the voltage were taken from network and fed to the analog pins of ATmega328P microcontroller.

\section{Cloth Glove}

A locally available Cotton glove was utilized on to which the flex sensors were mounted. It is shown in Figure 2.

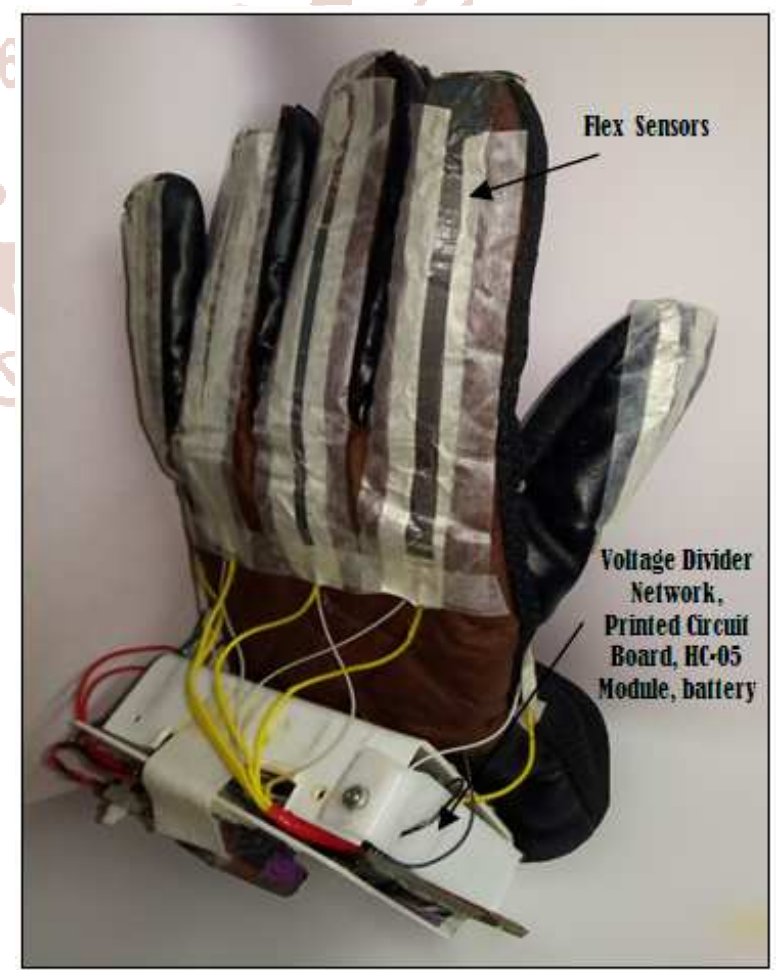

Figure 2: Practically Implemented Motion Detection Glove. 
$\begin{array}{ll}\text { E. } & \text { Power Supply } \\ \text { A. } & \text { For Transmitter }\end{array}$

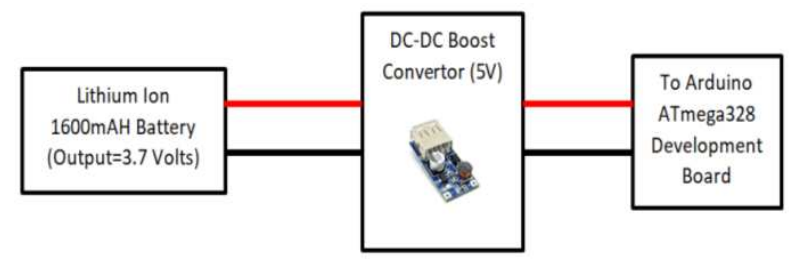

Figure 2: Transmitter Power Supply

Firstly a battery of 3.7 volts was used, now we required 5 volts for the microcontroller and the $\mathrm{HC}-05$ module, so we used a 3.7 volt to 5 volts DC-DC boost convertor and finally 5 volts were fed to both. Block diagram is shown in Figure 2.

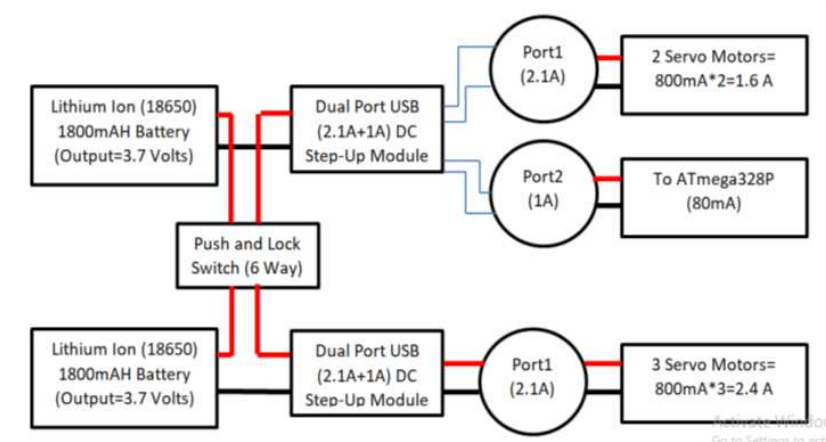

\section{B. For Receiver}

\section{Figure 3: Receiver Power Supply}

There were five servo motors which were to be driven. Each Servo motor consumes $800 \mathrm{~mA}$ so a single power supply was not sufficient so we came up with a solution to separate the supply to servo motor such that one power supply provides power to 3 servo motors and the other power supply provides power to remaining to servo motors and the microcontroller unit and HC-05 module. Block diagram is shown in Figure 3.

\section{Designing The Exoskeleton Glove}

This Part will be Exoskeletal Glove which would be worn in hand of the patient claiming physiotherapy exercises. Various parts were designed such as Fixed Clip, Variable Clip, Spring Tensioning mechanism, servo base, and variable palm consoler.

\section{A. $\quad$ Fixed Clip}

Each Finger has two joints, namely distal interphalangeal and, proximal interphalangeal joints, this fixed clip is designed to act as a permanent clip placed on the topmost part of the finger which will be usefull in simulating the distal interphalangeal joints. It is shown in Figure 4.1

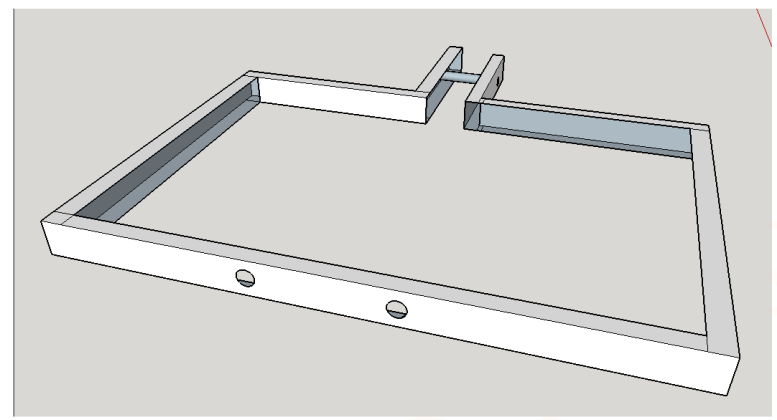

Figure 4: Fixed Clip

It will be useful in simulating the distal interphalangeal joints. Two holes are made so that a string could be attached and the servo motor can move the fingers.

\section{B. Variable Clip}

This clip is designed to simulate the proximal in terphalangeal joints of every finger. Here a two way cylindrical hole was made so that the stretch ability could be achieved else it would have remained at one position. It is shown in Figure 5.

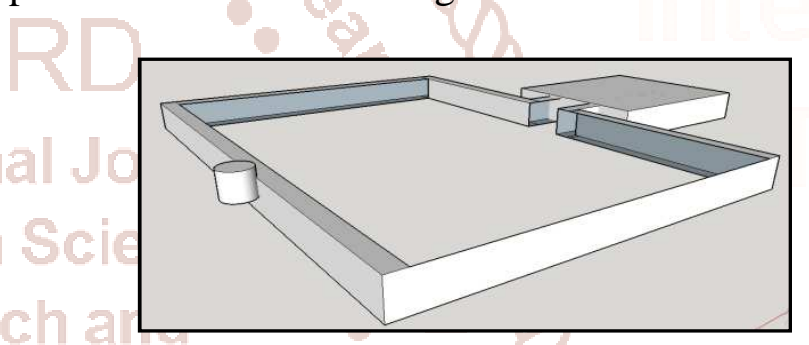

Figure 5: Variable Clip

\section{Spring tensioning mechanism}

This mechanism is used for applying stress on the finger. We are using spring which is a medium for applying stress on the finger. Spring is connected on each of the finger through fixed clip.

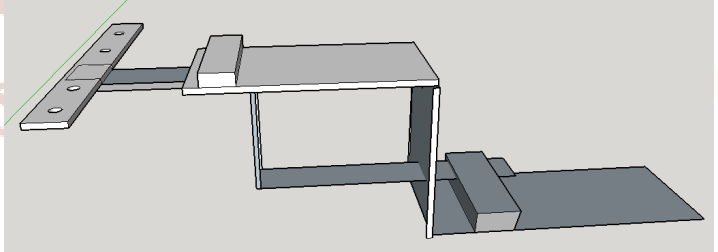

Figure 6: Spring tensioning mechanism

As shown we designed a mechanism such that we can control the area on to which stress is to be applied. It is shown in Figure 6.

\section{Servo Base}

This base was designed to mount the servo motors in a direction suitable for pulling the fingers. Each servo 
motor shaft was connected to through the fixed clip and variable clip. Servo Base is shown in Figure 7.

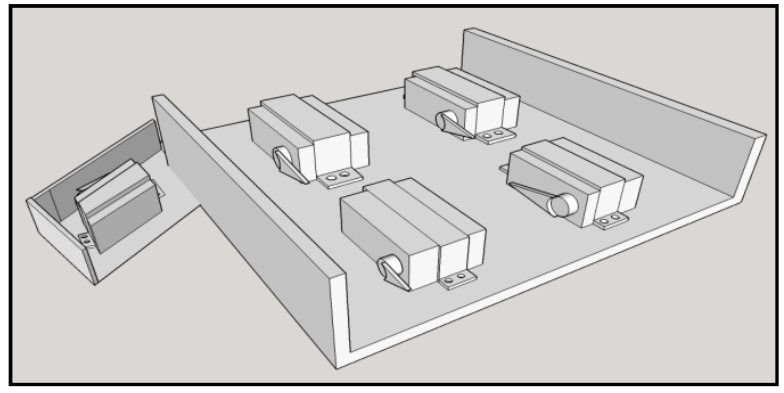

Figure 7: Servo Base

\section{E. Whole Structural View}

In order to arrange the whole setup this structure was designed. Various Indications are shown in Figure 4.5 .

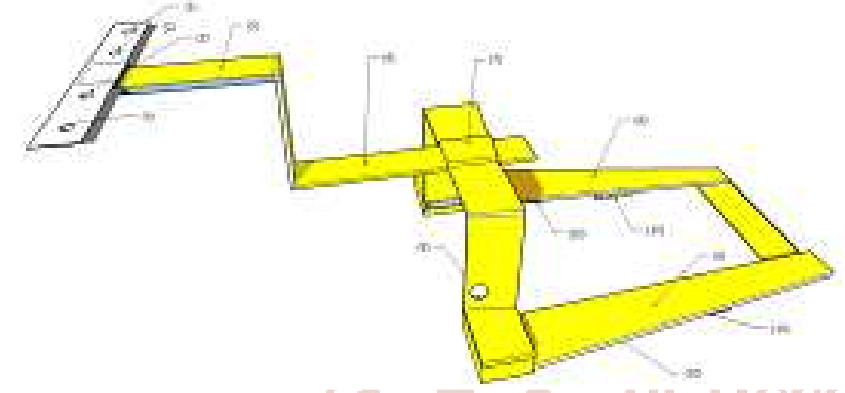

Figure 8: Whole Structural View

Various indications are shown in Table-I below

Table 1: Corresponding details of indications in Figure 8

\begin{tabular}{|c|c|}
\hline Indication & Label \\
\hline 1 & Spring attachment for index finger. \\
\hline 2 & Spring attachment for middle finger. \\
\hline 3 & Spring attachment for ring finger. \\
\hline 4 & Spring attachment for little finger. \\
\hline 5 & Movable spring stand. \\
\hline $5-6-7$ & $\begin{array}{c}\text { Variable stand for moving spring } \\
\text { tensioning mechanism. }\end{array}$ \\
\hline 8 & $\begin{array}{c}\text { Servo base Structure for moving the } \\
\text { fingers. }\end{array}$ \\
\hline 9 & Spring attachment for thumb. \\
\hline 10 & Assistive gripper for Servo Base. \\
\hline
\end{tabular}

\section{F. Practically Implemented Exoskeletal Glove}

This is the practically implemented exoskeletal glove. As seen in the Figure 4.7 below springs were attached to each finger and the spring tensioning mechanism could be displaced by $9 \mathrm{~cm}$.

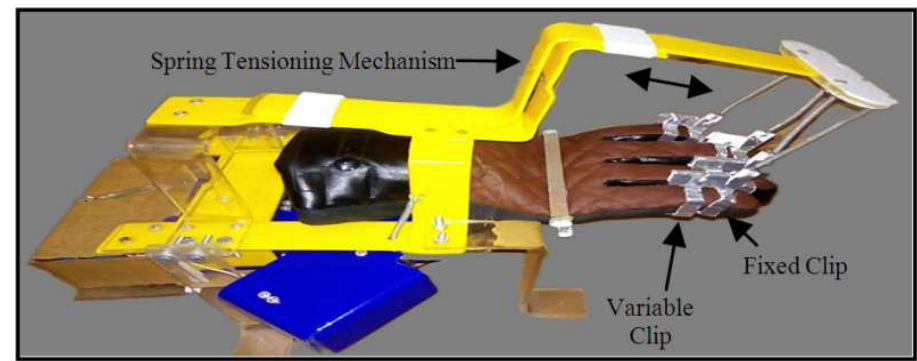

Figure 9: Practically implemented Exoskeletal Glove

\section{Results and Discussions}

There are so many things we can do with our fingers and hands without even thinking about it and if each of these movements is accompanied by pain and discomfort, life becomes very difficult. Some conditions can rectify themselves over time and some need expert attention from a physiotherapist. So below are some of the basic exercises which can be performed using Hand Rehabilitation Glove.

\section{A. $\quad$ Fist Stretch \& Release}

Make a gentle fist with your thumb wrapped across the fingers and hold it for 30-60 seconds. Then release and spread your hands wide [4]. It is shown if Figure 10.

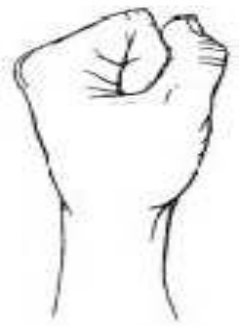

Figure 10: Fist transition

Try to do this at least four times with each hand. This is the first step in which our designed mechanism will create stress on finger.

\section{B. Claw Transition}

Another exercise to help with motion range is the claw stretch. Hold out the hand in front of you with the palm facing up. Bend over your fingertips to touch the base of each finger joint -hence the claw - and hold this for 30-60 seconds. Release the hand and then repeat at least four times [4].

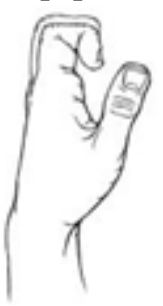

Figure 11: Claw transition 


\section{Thumb flexion/extension}

Begin with your thumb positioned outward \& move the thumb across the palm and back to the starting position [4].

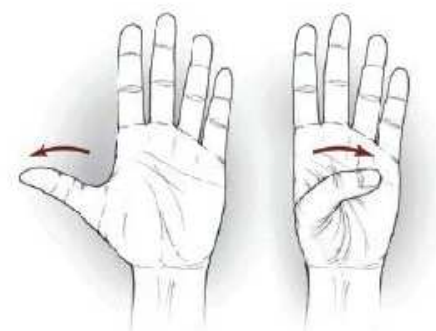

Figure 12: Thumb flexion/extension

Our Prototype is based on robotic orthosis which could be used for,

1. Radial nerve injuries

2. Ulnar nerve Injury (Little Finger, Middle Finger etc.)

3. Carpel Tunnel Syndrome (Excessive keyboard typing)

\section{References}

1. E. F. Shaw Wilgis, "The Wonder of the Human Hand" JHU Press, 10-Sep-2014.

2. Daniel Hunt, "Industrial Robotics Handbook" Chapter 2, Industrial Press Inc., 1983 H. H. Crokell, "Specialization and International Competitiveness," in Managing the Multinational Subsidiary, H. Etemad and L. S, Sulude (eds.), Croom-Helm, London, 1986. (book chapter style)

3. M. Atul. Doshi, Sagar. J. Parekh, Dr. Mita Bhowmick, "Wireless Robotic Hand using Flex Sensors", International Journal of Scientific \& Engineering Research (IJSER), Volume 6, Issue 3, Pg. No. 1471-1474, 2015

4. http://www.thephysiocompany.com/blog/handand-finger-exercises [Accessed: April. 12, 2004].

5. https://www.webmd.com/a-to-z-guides/brokenhand\#1 [Accessed: Oct. 15, 2017].

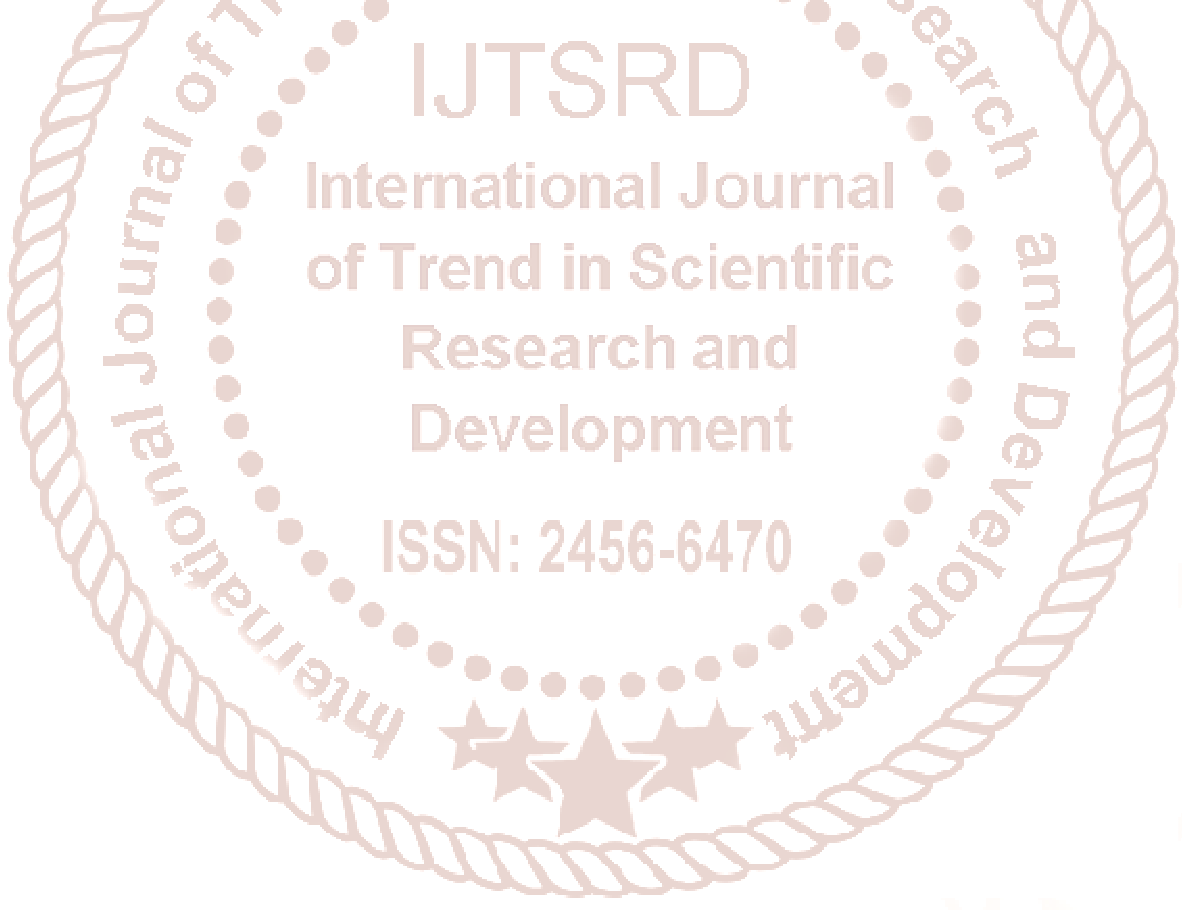

